\title{
Tuning The Quantum Yield of Fluorescent 2,5-Disubstituted-1,3a,6a-triazapentalene
}

\section{Key words}

fluorescent dyes

triazapentalenes

one-pot synthesis

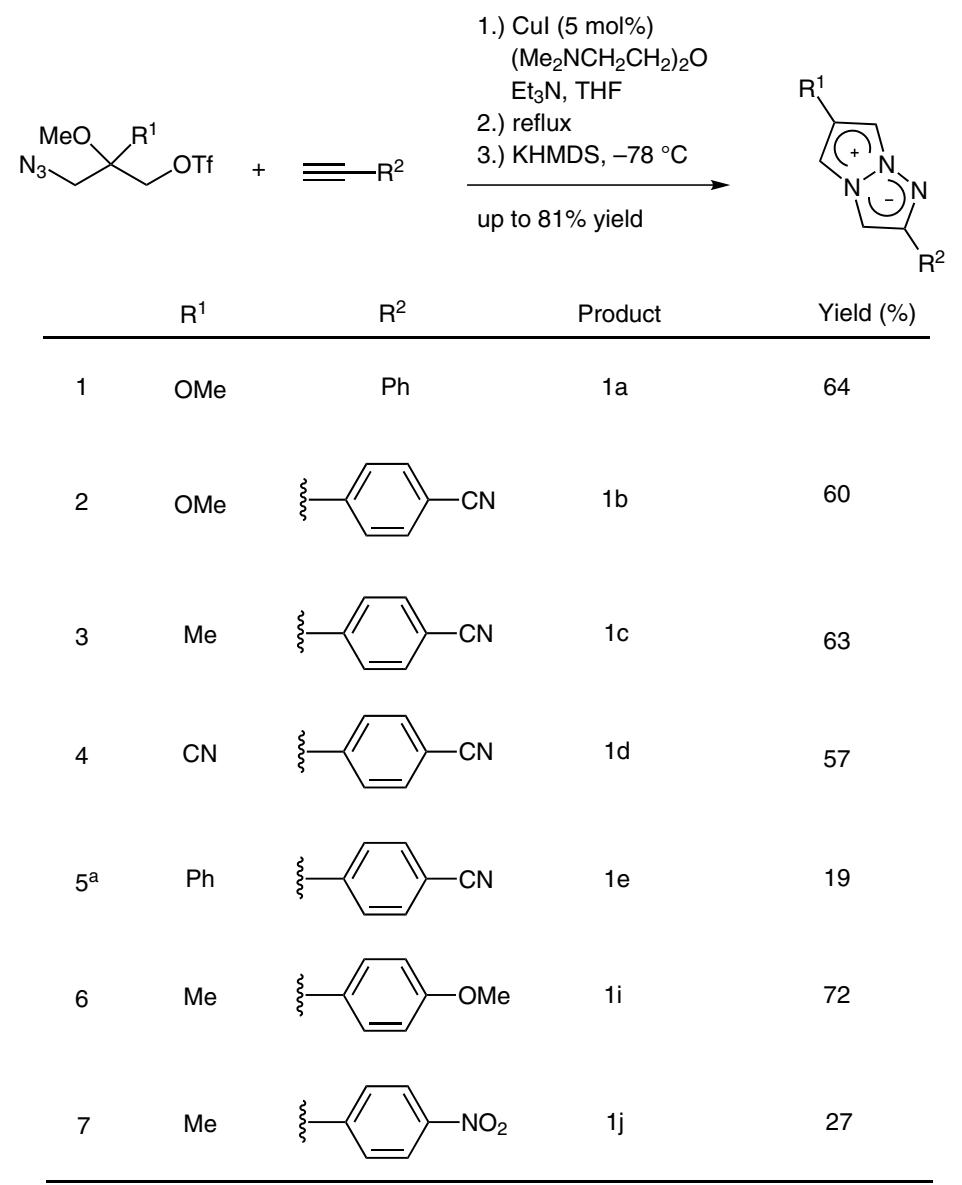

a The triflate was generated in situ and used without purification.

Significance: Rational design of organic molecules with improved photo-physical properties, such as high quantum yields and tunable fluorescence wavelength, is of great interest in modern science and technology. In this paper, the authors report a one-pot synthesis of 2,5-disubstituted1,3a,6a-triazapentalenes. By a cascade sequence utilizing a copper(I)-catalyzed 1,3-dipolar cycloaddition followed by intramolecular cyclization and elimination, the authors managed to obtain the desired 1,3a,6a-triazapentalene skeleton.
Comment: The authors report the synthesis of a series of 2,5-disubstituted-1,3a,6a-triazapentalenes. These novel compounds allowed the authors to probe the effects of electron-donating and -withdrawing substituents on the photo-physical properties of 1,3a,6a-triazapentalene derivatives. Introduction of substituents in the 5-position led to a dramatically increased quantum yield. A correlation between the Hammet $\sigma_{\mathrm{p}}$-value of the $\mathrm{R}^{2}$-substituent and the quantum-yield tendency could furthermore be estimated.

sYNFACTS Contributors: Timothy M. Swager, Jens B. Ravnsbæk 